\title{
RESTAURACIÓN DE LA CUBIERTA DEL TEATRO MUNICIPAL DE ALMAGRO. CASTILLA-LA MANCHA, ESPAÑA
}

\author{
(RESTORATION OF THE ROOF ON THE TEATRO MUNICIPAL OF ALMAGRO. \\ CASTILLA-LA MANCHA, SPAIN)
}

Francisco Jurado Jiménez, arquitecto

Fecha de recepción: 4 - VIII - 93

\section{RESUMEN}

Trata este artículo sobre las obras de restauración recientemente llevadas a cabo en la cubierta del Teatro Municipal de Almagro, bello ejemplo de la arquitectura teatral del s. XIX.

Se plantea la recuperación de las atrevidas formas estructurales de madera de la cubierta que, con muy pequeñas escuadrías, salvan luces muy importantes.

\section{SUMMARY}

This article deals with the restoration work recently performed on the roof of the Teatro Municipal of Almagro, a beautiful example of the theathe architecture of the XIX $c$.

What is proposed here is the retrieval of the daring wooden structural forms of the roof which save very important spans with very small scantling.
Informe sobre las cerchas de la cubierta (enero 1990)

A consecuencia de la aparición de unas fisuras en el interior del teatro se descubrieron unas deformaciones alarmantes en las piezas de madera de la estructura, bajo la cubierta existente sobre el patio de butacas.

Efectivamente, una de la cerchas presentaba una deformación (Fig.1) que allí mismo nos pareció totalmente inexplicable, puesto que, ni el peso propio de la cubierta, ni el de una lámpara colgada casi directamente de esta cercha, nos pareció capaz de inducir esa deformación. Sólo nos pareció posible que ésta apareciera por un hinchamiento debido a la humedad, pero no existían huellas recientes de penetración de agua.

La pieza de madera deformada estaba levantando ostensiblemente el faldón de la cubierta, sobrecargando a las cerchas colindantes. Y una de las cer- chas colindantes tenía una deformación muy similar, pero en el lado contrario, y había sido reparada hace tiempo con pletinas metálicas.

Casi todos los nudos de las cerchas presentaban movimientos que han provocado desgajamientos y aperturas de las piezas atravesadas por pernos metálicos.

Utilizando un programa de análisis matricial, se analizaron varios casos, tanto de geometría como de cargas, para poder evaluar el comportamiento de la estructura de la cubierta.

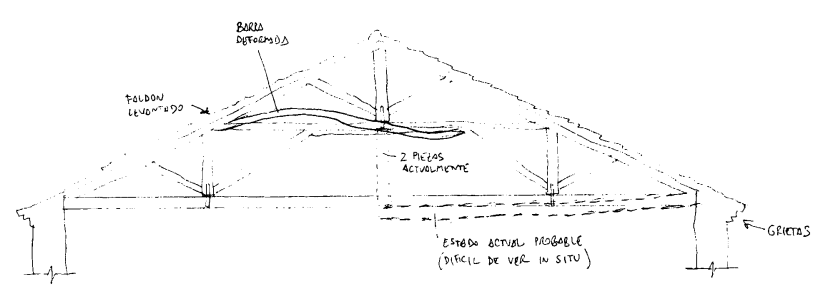

Fig. 1.- Estado actual cercha. 


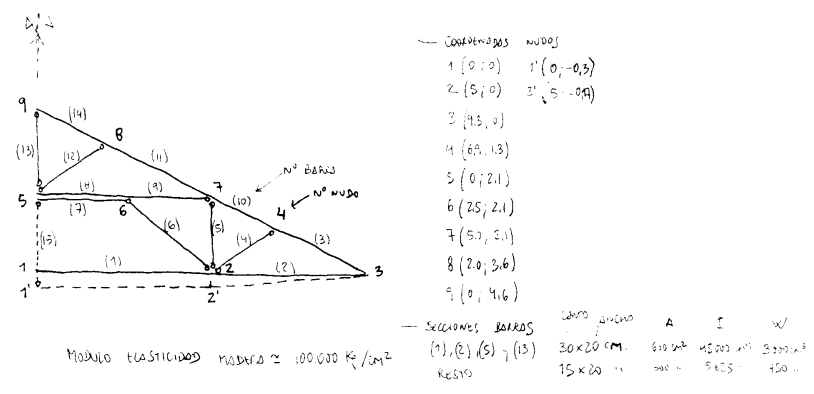

Fig. 2.- Modelo estmictural analizado.

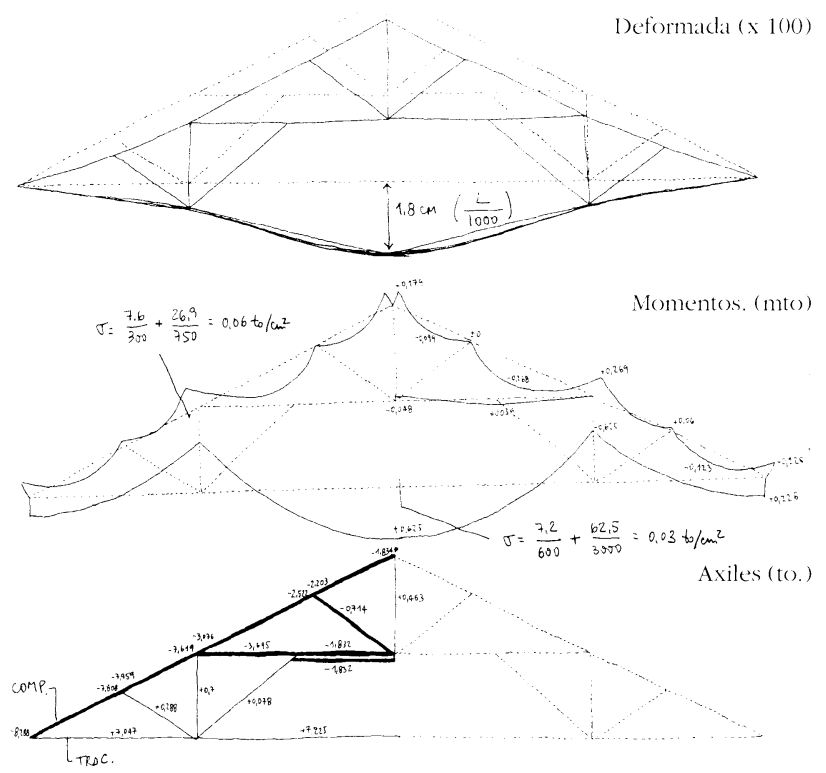

Fig. 3.- Estructura original con carga vertical.
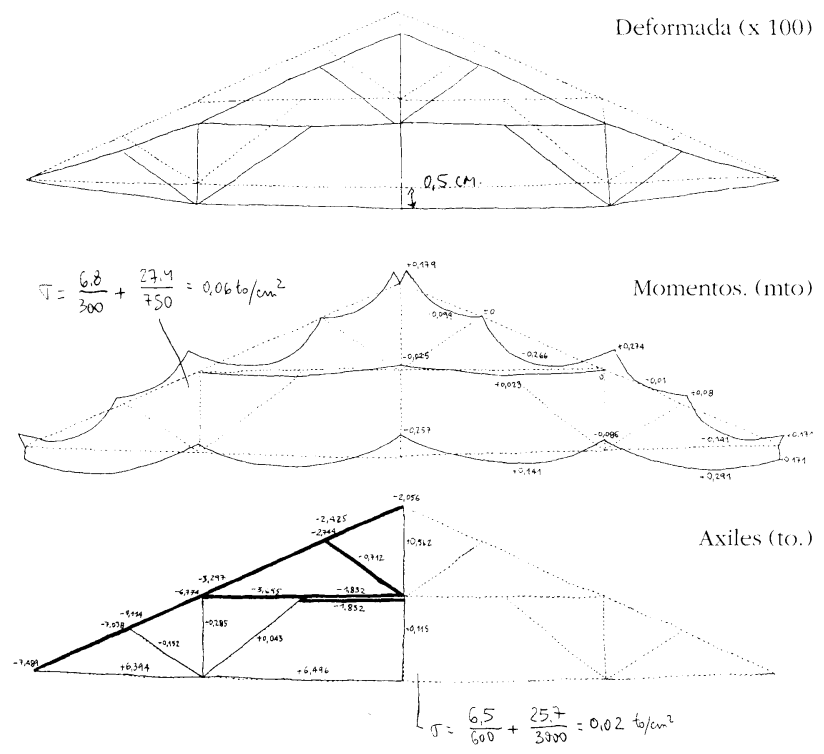

Fig. 4.- Estructura actual con carga lertical.
En la figura 2 se representa el modelo estructural analizado. La barra 15, al ser un elemento anadido a la cercha original, aparece en unos casos sí y en otros no.

Los casos analizados fueron los siguientes:

\section{1.- Cercha original sometida a carga vertical}

Corresponde a la cercha sin la barra 15 , cargada con un peso de $50 \mathrm{~kg} / \mathrm{m}^{2}$ en el tirante inferior (el falso techo) y con $165 \mathrm{~kg} / \mathrm{m}^{2}$ en los faldones (el peso propio de los mismos con la teja y nieve).

Los resultados se resumen gráficamente en la figura 3 , donde se observa que, para las secciones y cargas supuestas, las máximas tensiones normales resultantes son $60 \mathrm{~kg} / \mathrm{cm}^{2}$ en los pares y $30 \mathrm{~kg} / \mathrm{cm}^{2}$ en el tirante inferior.

Suponiendo que la madera resista alrededor de 100 $\mathrm{kg} / \mathrm{cm}^{2}$, es obvio deducir cuánto vale el coeficiente de seguridad en cada caso.

\section{2.- Cercha actual sometida a carga vertical}

Corresponde a la cercha con la barra 15 y el tirante inferior deformado, como parece apreciarse in situ. $\mathrm{Al}$ igual que antes, se consideran cargas de $50 \mathrm{~kg} / \mathrm{m}^{2}$ en el tirante inferior (el falso techo) y de $165 \mathrm{~kg} / \mathrm{m}^{2}$ en los faldones.

Los resultados se resumen gráficamente en la figura 4 , donde se observa que, para las secciones y cargas supuestas, la introducción de la barra 15 mantiene la máxima tensión normal de $60 \mathrm{~kg} / \mathrm{cm}^{2}$ en los pares, bajando a $20 \mathrm{~kg} / \mathrm{cm}^{2}$ en el tirante inferior. Pero el efecto más notable es que reduce la flecha del tirante más de 3 veces, por lo que debió de ponerse cuando la flecha del mismo, debido a los fenómenos de fluencia, empezara a alarmar visualmente.

\section{3.- Cercha actual sometida a la carga de la lámpara}

Corresponde a la cercha con la barra 15 y el tirante inferior deformado.

Se ha considerado una carga puntual de $1.000 \mathrm{~kg}$ en el nudo 5, correspondiente al máximo efecto (incluso con consideraciones dinámicas) que puede producir la lámpara colgada de ese nudo. 
Los resultados se resumen gráficamente en la figura 5 , donde se observa que, para las secciones y cargas supuestas, los efectos de esta carga (que habría que adicionarlos a los de las cargas consideradas en la hipótesis anterior) son bastante inapreciables, puesto que introducen tensiones de $3 \mathrm{~kg} / \mathrm{cm}^{2}$, o menores, y la deformación es inapreciable a simple vista (flechas menores de $1 \mathrm{~mm}$ sólo podrían medirse con flexímetros sujetos a puntos fijos).

\section{4.- Cercha original sometida a binchamiento}

Aunque hemos intentando analizar la cercha actual desdoblando el nudo 6 , de modo que modelizásemos el despegue que ha existido entre las barras $7 \mathrm{y}$ 8-9, problemas de condicionamiento numérico en la matriz de rigidez de la estructura impiden una resolución correcta del sistema de ecuaciones.

Hemos optado, por tanto, por analizar el efecto de un hinchamiento de un 1 por mil en las barras 8 y 9 (una única pieza en la realidad) sobre el modelo de la cercha original. Los resultados se resumen gráficamente en la figura 6 , donde se observa que, para las secciones y deformación impuesta, la deformación en la estructura es muy similar a la que se ve in situ, sólo que, en la realidad, la deformación es unas 20 veces mayor (en lo que concierne a la zona despegada).

Por esta similitud pasamos a analizar el caso siguiente, más acorde con la situación real.

\section{5.- Cercha en la situación actual, con carga vertical e binchamiento.}

Corresponde a la cercha con la barra 15 , y el tirante inferior deformado.

Se han considerado las cargas verticales correspondientes a la cubierta y al falso techo inferior, y se ha añadido también el efecto de un hinchamiento en la barra 9 (el introducirlo en la barra 8 lleva consigo un efecto contrapuesto en la barra 7, pegada a ella en el modelo, aunque no en la realidad).

El valor del hinchamiento introducido en la barra 9 ha sido del $2 \%$. Los resultados se resumen gráficamente en la figura 7 , donde se observa que, para las secciones y cargas supuestas, la deformación es muy fuerte (se ha dibujado 10 veces menor que en el resto de los casos), así como también lo son las solicitaciones.
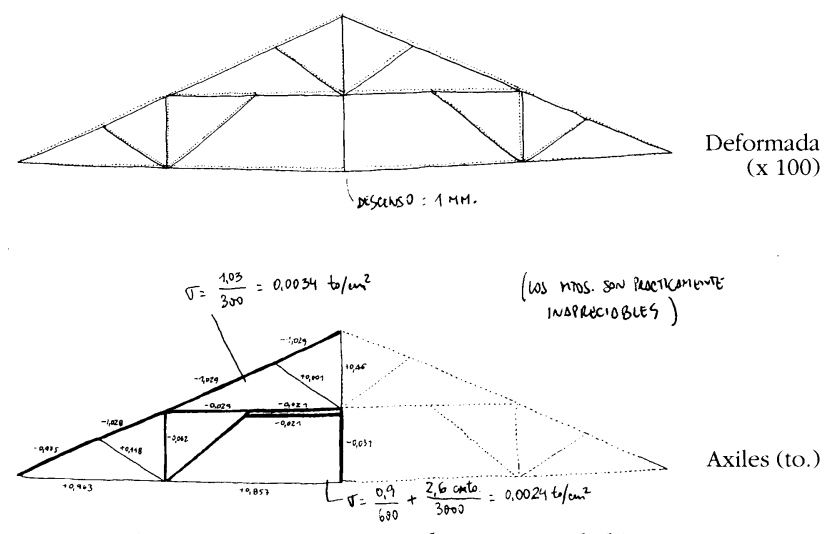

Fig. 5. - Estructura actual con carga de lámpara.
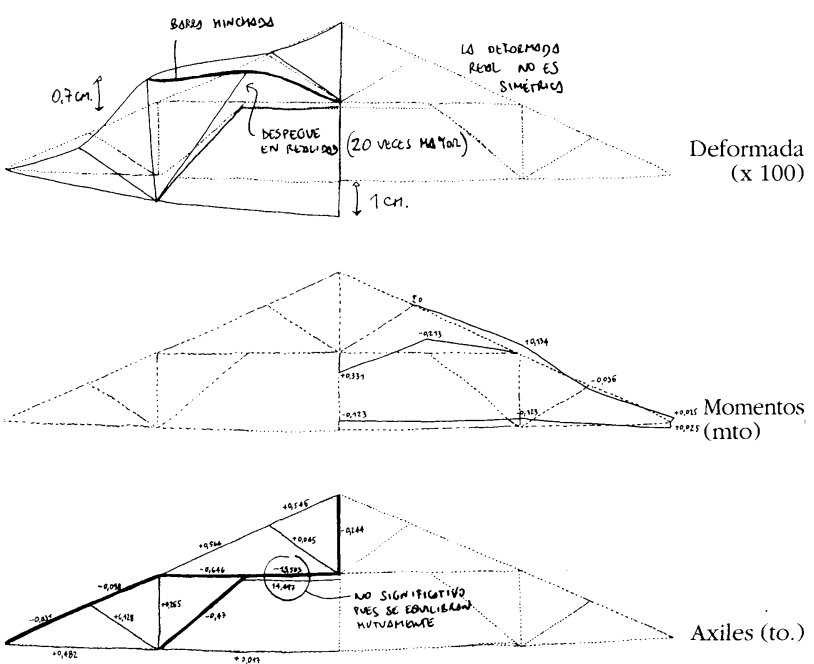

Fig. 6.- Estructura original con binchamiento barras.

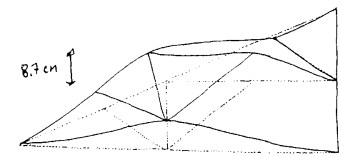

Deformada (x 10)
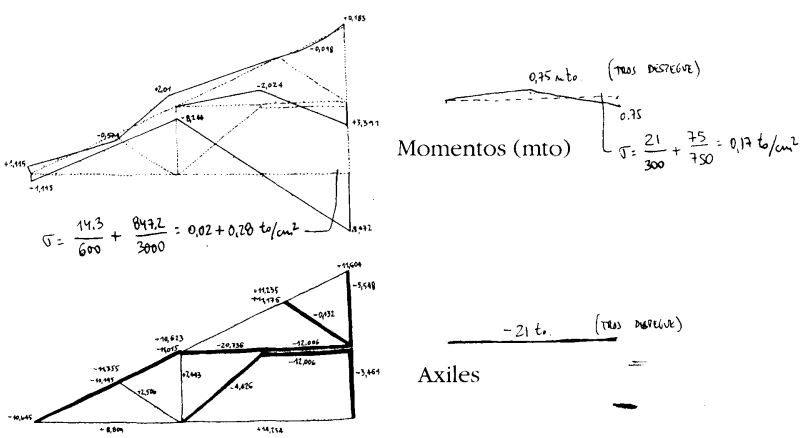

Fig. 7.- Cercha actualmente. 


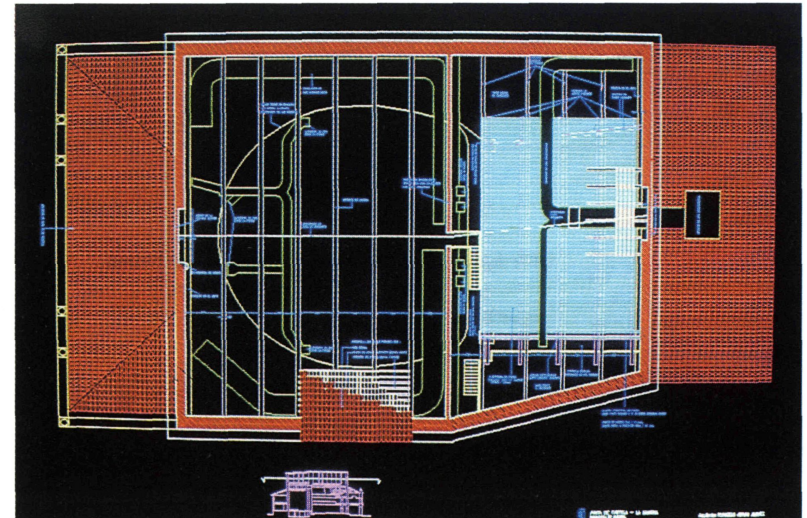

Planta del bajo cubierta. El círculo que se percibe bajo las formas de madera corresponde a la platea. El tupido entramado de la derecha se corresponde con una nueva estructura metálica, que se construyó para la zona de los "peines" de los decorados.

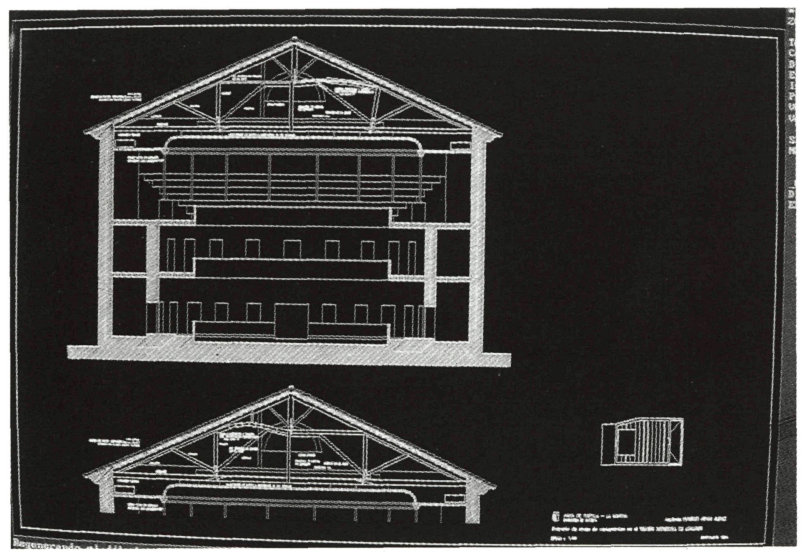

Sección transversal por la platea antes de la restauración.

De todos modos, hay que hacer ciertas salvedades debido a que, en la realidad, existe un despegue, como ya hemos dicho, en el nudo 6 y el comportamiento no es simétrico.

Por aproximarnos más a la situación real de la barra 8-9 añadimos manualmente el efecto del despegue existente citado y, según se ve en la figura, la pieza 8-9 está sometida a tensiones máximas del orden de $170 \mathrm{~kg} / \mathrm{cm}^{2}$, valor que, dependiendo del estado y calidad de la madera, pueda haber agotado la seguridad ante la rotura.

También parece que el tirante inferior está sometido a unos flectores que le producen tensiones mayores de $250 \mathrm{~kg} / \mathrm{cm}^{2}$, algo creíble en función de cómo varíe el modelo estructural con el despegue en el nudo 5 .

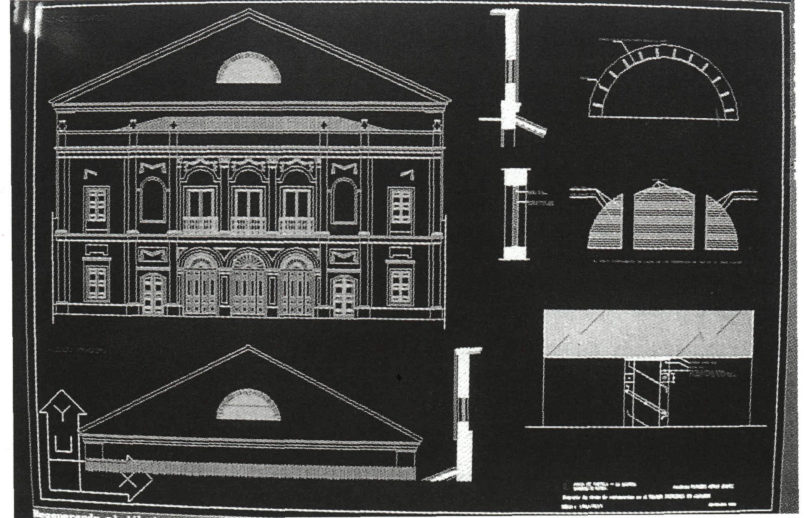

Plano de restauración de fachadas con recuperación de los óculos de la cubierta.

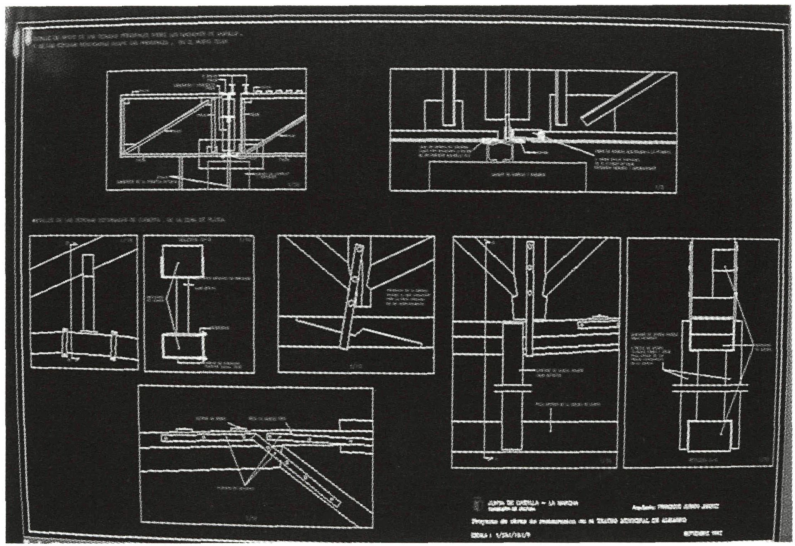

Detalles de uniones con deformaciones y roturas en la zona superior en la estructura metálica de los peines, y abajo en las formas de madera.

Las conclusiones del informe eran las siguientes:

1) La cercha original tiene un diseño adecuado para la geometría y cargas que debe solventar, teniendo una seguridad ante las tensiones normales máximas mayor de 2 .

2) Es probable que al deformarse excesivamente con el tiempo el tirante inferior se añadiera la barra 15 que, prácticamente, lo único que hacía era colgar el tirante inferior, evitando que su deformación progresase.

3) El peso de la lámpara no es nada significativo en su acción sobre la cercha.

4) Parece que la deformación que actualmente posee la barra 8-9 de la cercha únicamente parece 


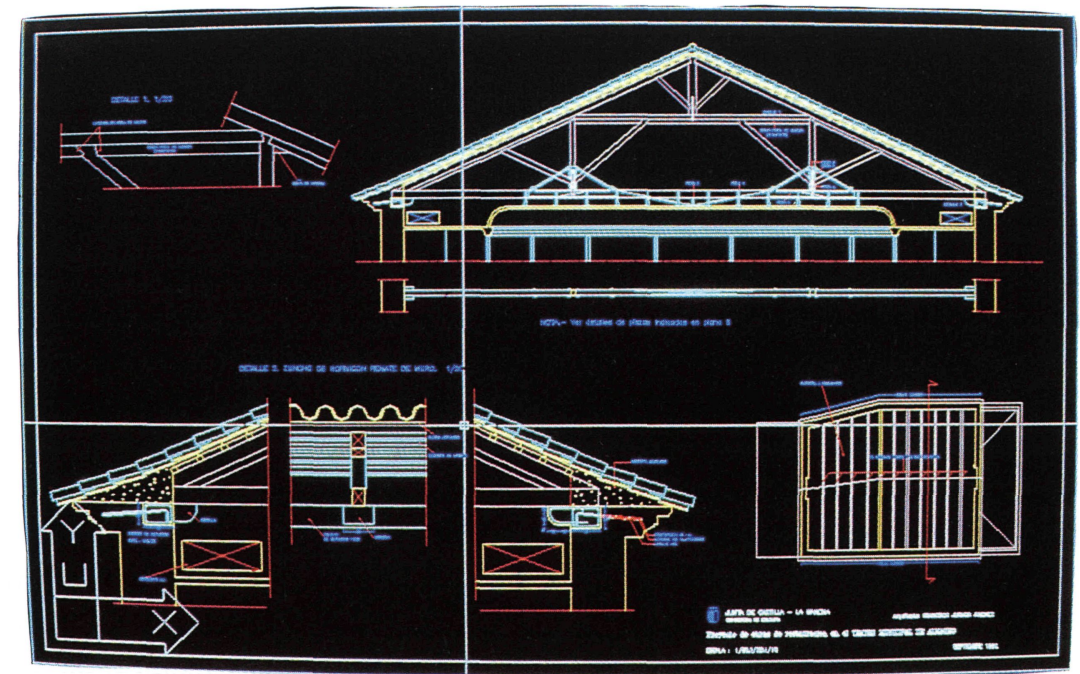

Plano de restauración de la cubierta con las formas de madera reforzadas, y detalles de los nuevos apoyos de las formas de madera sobre los muros.

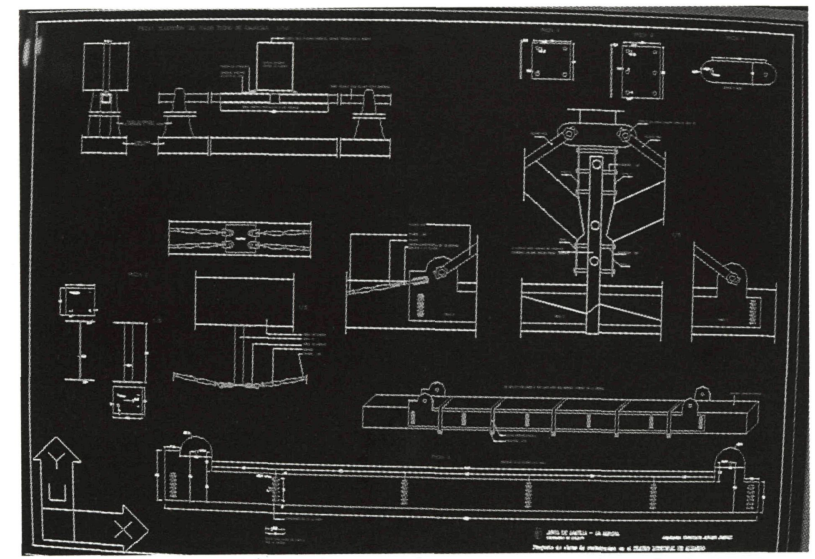

Detalles de las reparaciones y refuerzos de las formas de madera con pletinas y tirantes metálicos.

explicable por un aumento de longitud de la misma, debido a alteraciones en su contenido de humedad (como parece haber ocurrido en otra ocasión en la cercha colindante).

En efecto, la madera tiene una variación despreciable de longitud con la humedad en el sentido de las fibras, pero no así en dirección perpendicular, donde se puede llegar a variaciones del 10 y $15 \%$. Depende de cómo se haya cortado la pieza deformada, le pillará en determinadas zonas las fibras de modo transversal a su longitud, produciéndose efectos parecidos al analizado.

El que se nos afirme que no hay penetración de agua en la cubierta es relativamente verosímil, ya que, para que se produzca el aumento de volumen longitudinal del 2\% considerado, teniendo

(c) Consejo Superior de Investigaciones Científicas Licencia Creative Commons 3.0 España (by-nc)

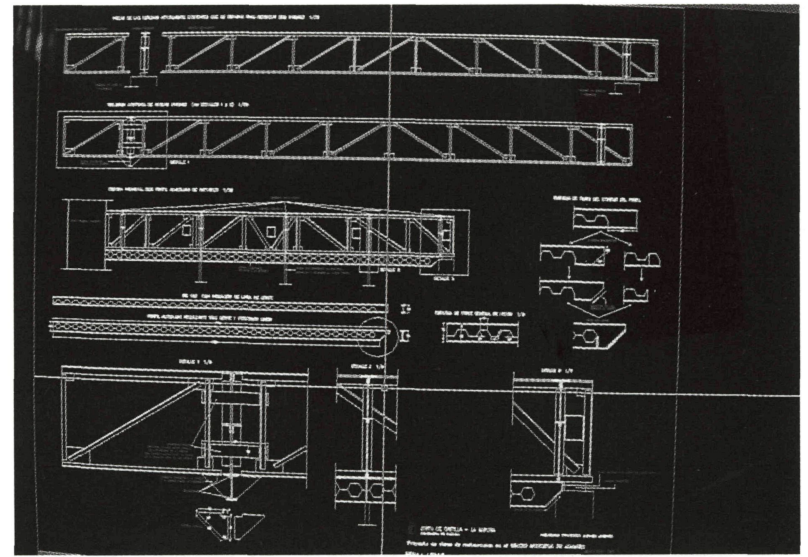

Detalles de las reparaciones y refuerzos de las cerchas metálicas de los peines.

en cuenta la higroscopicidad de la madera, podía ser suficiente un aumento de la humedad relativa del interior de la cámara.

5) El último caso analizado sólo debe entenderse como una aproximación a la realidad, mediante un modelo de comportamiento teórico, por lo que no se deben extraer conclusiones al pie de la letra, ya que la geometría real está totalmente alterada, así como la mayoría de las uniones.

En cualquier caso, las tensiones a las que se ve, probablemente, sometida la madera actualmente, son del todo inseguras.

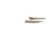

Por todo lo expuesto, nuestras recomendaciones fueron las siguientes: 
1) Reparar la cercha objeto del informe cortando la barra 8-9 tras un adecuado apeo a la vista de su comportamiento en la estructura original, sustituyéndola por pieza de similar escuadría y secada hasta una humedad similar a la del interior de la cubierta.

2) Efectuar reparaciones locales en los nudos y piezas desgajadas mediante pletinas metálicas y pernos o tornillos.

3) Efectuar una adecuada rehabilitación de la cubierta que contemple el repaso de toda la estructura principal de cerchas y la secundaria de correas o parecillos; que contemple una completa impermeabilización de los faldones y una adecuada ventilación del bajo cubierta; que zunche la cabeza de los muros que tienden a abrirse, apoyando adecuadamente las cerchas y evitando los agrietamientos en las cornisas.

\section{Situación inmediata a la realización del proyecto}

Tras el informe resumido anteriormente transcurrieron dos años sin que se efectuase ningún tipo de reparación y se nos volvió a llamar porque aparecieron fisuraciones importantes en el falso techo de escayola que cuelga de la cubierta de madera, así como un incremento apreciable de todos los desgajamientos de uniones de la madera que ya existían. Sí que se había efectuado, sin embargo, una reparación de urgencia en las dos cerchas que presentaban la deformación tan exagerada y que observábamos en el informe del año 1990. Dicha reparación consis-

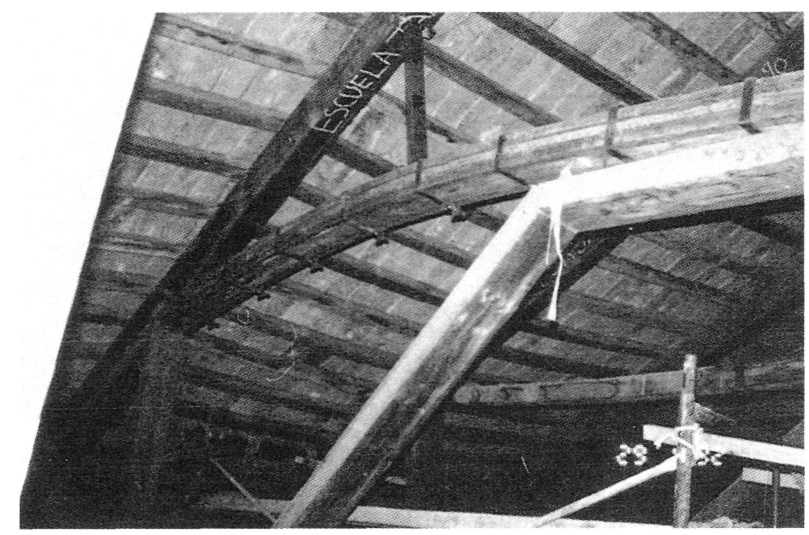

Aspecto parcial de una de las cerchas de madera deformada con un intento de reparación mediante bridas metálicas.

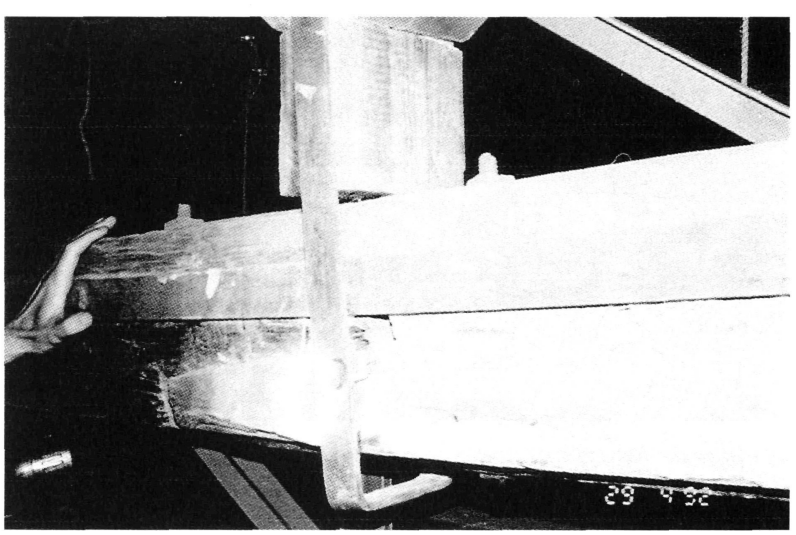

Detalle de una unión en "rayo de Jupiter" en un tirante dispuesta al revés, de modo que se separaría con la tracción si no fuese por la brida metálica longitudinal.
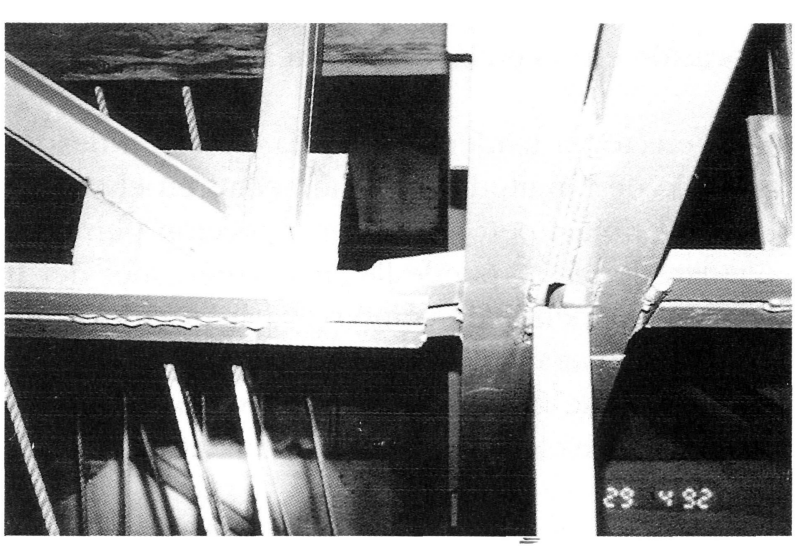

Detalle del "apoyo" de una cercha metálea secundaria sobre la principal mediante un trozo de angular, en la zona de los "peines".
Detalle de unión similar a la anterior con el "rayo" correctamente dispuesto (no es necesaria la brida metálica), pero con un movimiento diferencial del tirante respecto al pendolón superior que ha desgajado a éste. 
te en colocar unas bridas metálicas que fijan las piezas curvadas en la posición deformada que adquirieron en ese momento.

Sin embargo, los movimientos en la estructura de la cubierta no se detuvieron con esta reparación, ya que al ceder los tirantes, inferiores unidos a los pendolones mediante estas uniones que estaban desgajadas, han ido aumentando y ha provocando la fisuración del falso techo de escayola.

Observando también -esta vez con más detenimiento- toda la estructura metálica de cerchas y piezas que se colocaron en la restauración del año 1988 para sujetar los peines de la escena, se descubrieron unas uniones absolutamente peligrosas, puesto que al parecer las nuevas cerchas metálicas debieron quedar cortas en su colocación y se hizo una serie

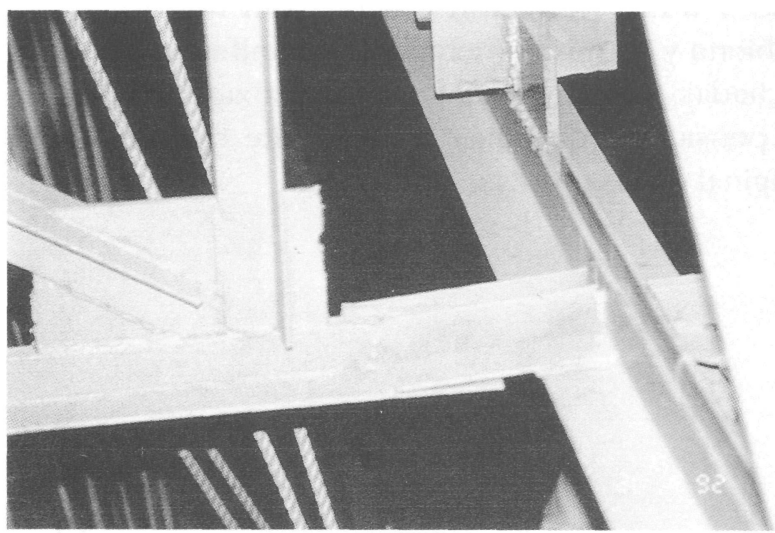

Misma unión que la foto anterior, vista desde arriba.

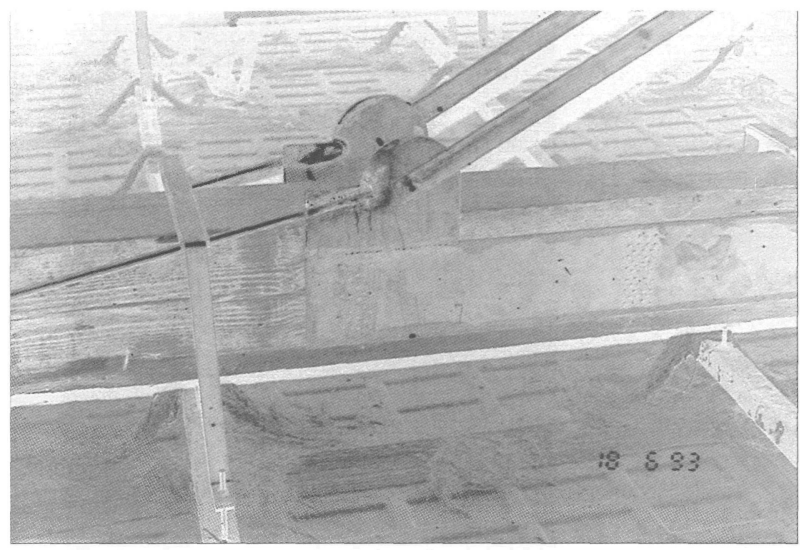

Detalle del refuerzo metálico de las formas de madera. Obsérvense las bandas de clavos que conectan las pletinas laterales con la sección de madera, así como las nuevas sujeciones de la escayola sobre la platea.

(c) Consejo Superior de Investigaciones Científicas Licencia Creative Commons 3.0 España (by-nc) de uniones por medio de casquillos soldados hasta llegar al punto de apoyo, de modo que, como puede observarse en las fotografías que se adjuntan, ya había incluso deformaciones debidas a flexiones en elementos que tenían que estar directamente trabajando a compresión o a tracción, como corresponde a una estructura triangulada. Tanto es así, que nos pareció mucho más grave incluso estas soluciones que las deformaciones de las cerchas de madera.

\section{Solución adoptada}

La solución adoptada en lo concerniente a la estructura de madera se basa en los resultados numéricos obtenidos en nuestro informe del año 1990.

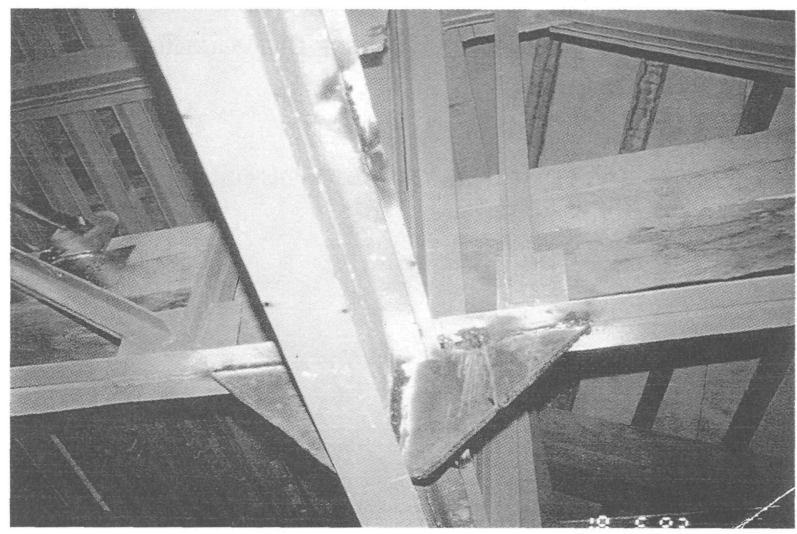

Reparación del apoyo de la cercha metálica mediante un suplemento del cordón inferior con dos cartelas que recogen las cargas de los montantes.

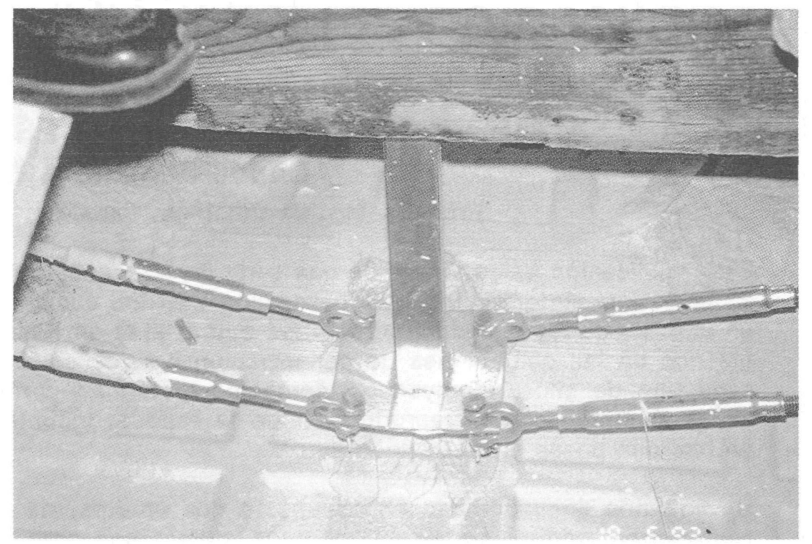

Detalle del pendolón de refuerzo a flexión del tirānte de madera con los tensores metálicos. 
Nuestro criterio de intervención en este caso es el de mantener la estructura principal de la cubierta, que creemos correctamente diseñada. Por tanto, se van a mantener las cerchas no sólo como están sino eliminando el montante central que se colocó en época más contemporanea para mejorar la flexión asociada al tirante inferior.

Como consecuencia del mencionado informe que hicimos en 1990, vemos que, prácticamente todas las cerchas, en su estado original, están perfectamente diseñadas, sólo que tienen algunos puntos débiles, para lo cual introducimos una pieza de madera que refuerza a compresión precisamente la pieza que se curvó, e introducimos unos elementos metálicos que aumentan sensiblemente la capacidad resistente a flexión del tirante inferior. Esta pieza, que tendería a trabajar como viga continua colgada de los pendolones, resulta que tiene una unión en "rayo de Júpiter" (en algunos casos dispuesta al revés, sin posibilidad de que el tirante funcione como tal) justo debajo de los mismos, lo que ha provocado que actúe como una articulación, girando, partiéndose la unión y arrastrando al pendolón que ha provocado las roturas en esas uniones.

Las piezas de refuerzo que nosotros proponemos son, por un lado, unas platabandas que garantizan la continuidad del tirante trabajando a tracción; por otro lado una serie de redondos y pletinas que forman una armadura asociada a la madera, de modo que, al final, tenemos una forma de viga mixta (madera-acero) que funciona como viga continua y como tirante, sin ningún problema de deformaciones excesivas adicionales.

En lo concerniente a las cerchas metálicas, lo que hacemos es colocar suplementos de piezas metálicas de modo que puedan trabajar consecuentemente con el diseño de piezas trianguladas tal como se diseñaron, disponiéndose la perfilería necesaria para que puedan resistir los esfuerzos provocados por los descentramientos en los apoyos y así mismo se transmitan de un modo fiable las cargas a los mismos.

Aprovechamos también esta intervención en la cubierta para abrir los dos semióculos o huecos semicirculares que hay en ambos testeros, en ambos piñones triangulares de la cubierta, para que se produzca una ventilación efectiva del interior de la cubierta y, al mismo tiempo, los manifestamos en las fachadas, que presentan así un acabado más digno y, pensamos, que más consecuente con el diseño original de las mismas.

\section{publicación del ICCET/CSIC}

\section{ACUEDUCTOS ROMANOS EN ESPAÑA \\ Carlos Fernández Casado}

Prof. Dr. Ing. de Caminos, Canales y Puertos

Esta publicación se compone de una serie de artículos, publicados en la Revista "Informes de la Construcción", en los cuales se hace un análisis de los acueductos romanos que existen en España y el balance de las condiciones de conservación en que se encuentra cada uno de ellos, incluyendo referencias históricas y literarias. Se ha ilustrado con la reproducción de la valiosa documentación gráfica que posee el prestigioso autor.

Un volumen encuadernado en couché, a dos colores, de $21 \times 27$ centimetros, compuesto de 238 páginas, numerosos grabados, dibujos, fotos en blanco y negro y figuras de linea.

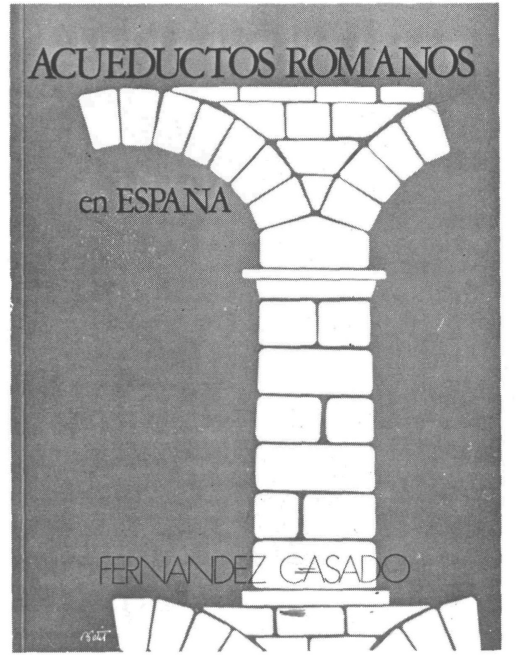

